\title{
CHANGES IN THE STIFFNESS OF THIGH MUSCLES IN THE LEFT AND RIGHT LIMBS DURING SIX WEEKS OF PLYOMETRIC TRAINING IN VOLLEYBALL PLAYERS
}

\author{
DARIUSZ MROCZEKIA, EDWARD SUPERLAKIA, MAREK KONEFAŁ'1A, KRZYSZTOF MAĆKAŁA2A, \\ PAWE€ CHMURA ${ }^{2 B}$, TOMASZ SEWERYNIAKIB, JAN CHMURA ${ }^{1 A}$ \\ ${ }^{1}$ University School of Physical Education in Wroclaw, Faculty of Sport Science, Department of Biological and \\ Motor Bases of Sports ${ }^{a}$, Department of Sports Communication and Management ${ }^{b}$, Wrocław, Poland \\ 2 University School of Physical Education in Wroclaw, Faculty of Physical Education, Department of Athletics \\ and Gymnastics ${ }^{a}$, Department of Team Sports Games ${ }^{b}$, Wrocław, Poland
}

\author{
Mailing address: Jan Chmura, University School of Physical Education in Wroclaw, Department of Biological and \\ Motor Bases of Sports, 35 Paderewskiego Street, 51-612 Wrocław, tel.: +48 71 3473141, fax: +48 713473397 , \\ e-mail: jan.chmura@awf.wroc.pl
}

\begin{abstract}
Introduction. Monitoring muscle stiffness in athletes can be a good method of assessing fatigue caused by high training loads, and the early detection of fatigue can help prevent the occurrence of micro-trauma in the muscles that can cause contusions. The research carried out by Wilson et al. [1] confirmed that an optimal level of muscle stiffness is significantly correlated with high muscle loads. The aim of the current study was to determine changes in muscle stiffness of the left and right thighs during six weeks of plyometric training (PT) in volleyball players. Material and methods. The study involved 16 volleyball players from the second-league Opole University of Technology Club (age $=21.12 \pm 1.66$ years, height $=191.62 \pm 5.73 \mathrm{~cm}$, and weight $=86.25 \pm$ $6.66 \mathrm{~kg})$ with at least five years of competitive experience $(7.5 \pm 2.44$ years $)$. Muscle stiffness was measured during three stages of the plyometric training using a MYOTON PRO device (Estonia). Results. An RM-ANOVA analysis showed a significant difference in the resting stiffness of the semitendinosus (posterior thigh) muscles of the left and right limbs before the plyometric training began, but no significant differences were found in the stiffness of these muscles in the fourth or sixth weeks of training. The results of the measurement performed for the anterior muscles of the thigh did not reveal a significant difference in the stiffness of the left limb compared to that of the right limb in subsequent weeks of training. Conclusion. The loads used in plyometric training in volleyball players caused a decrease in the differences in muscle stiffness between the left and right limbs, and in both limbs, adaptation trended towards an increase or a decrease in stiffness.
\end{abstract}

Key words: muscle parameters, jumping training in volleyball, Myoton PRO

\section{Introduction}

Preparing professional volleyball players for participation in competitions requires undertaking rational actions with respect to developing the players' motor, technical, and tactical skills. The modern training process has to make it possible for players to sustain very high loads during the match, since increasing fatigue may cause players to make errors during the game. Adequate planning of the training of volleyball players for competitions involves selecting specific methods, maintaining appropriate proportions between training loads, competition loads, and effective recovery, as well as administering adequate dietary supplementation.

One of the ways of preventing muscle overload in athletes is monitoring muscle stiffness, which can be a reliable parameter for assessing fatigue caused by high training loads. Detecting fatigue early can help prevent micro-trauma in the muscles which can lead to contusions. The research carried out by Wilson et al. [1] has confirmed that an optimal level of muscle stiffness is significantly correlated with increases in muscle load. In the current study, the volleyball players completed a six-week plyometric training (PT) programme during the preparatory period before the season, and the effects of this programme were assessed by measuring the increases or decreases in the level of stiffness of the thigh muscles in both lower limbs. Studies involving young healthy men with low or moderate levels of physical activity [2] have shown that the relative ratio between the stiffness of the anterior and posterior thigh muscles is approximately 1:1. It was found that asymmetry amounting to $15 \%$ increases the risk of injury [3]. In other studies, asymmetry in muscle stiffness was $6 \%$, and, as suggested by the authors [4], such results indicate that an asymmetry of stiffness parameters amounting to $10 \%$ may be evidence of abnormalities in muscle stiffness in young healthy males who do not practise sports. Other research has confirmed that muscle stiffness asymmetry for the quadriceps in healthy older men is not high, and the differences between the dominant and non-dominant limbs amount to approximately $2.5 \%$ for all muscle parameters [5].

Our innovative research into muscle stiffness in volleyball players can make it possible to use this parameter as a reliable predictor of fatigue caused by overtraining. Masi et al. [6] have shown that the objective measurement of the tone, tension, and 
mechanical properties of the muscles, such as their elasticity and stiffness, can allow for the effective identification of overload states and significantly reduce the risk of contusion. An increasing number of studies conducted with respect to this issue have confirmed that there is a need to monitor muscle stiffness in athletes who are exposed to high training loads [7-10].

The research conducted so far has sought to characterise the parameters of the thigh muscles in the same limb and thus to determine the risk of injury or to assess existing injuries. It seems that it is also important to compare the level of the stiffness of the thigh muscles in the left and right limbs in order to identify irregularities, but in order to do so, it is necessary to define the acceptable level of asymmetry between the two sides of the body. Determining the level of the stiffness of the thigh muscles may help to determine the threshold training load which if exceeded, can lead to the players sustaining microtrauma or contusions of the muscles.

The literature reviewed has not investigated athletes exposed to high training loads. Establishing the level of the stiffness of the thigh muscles and the degree of asymmetry between the left and right limbs not only is important from a cognitive point of view but also has practical applications, since it can make it possible to improve the planning and implementation of the training process and effectively solve problems related to overtraining in athletes. The aim of the current study was to determine changes in muscle stiffness in the left thigh compared to the right thigh during six weeks of plyometric training in volleyball players.

\section{Material and methods}

The study involved 16 volleyball players of the Opole University of Technology Academic Sport Union Club (AZS Politechnika Opolska). The subjects (age $=21.12 \pm 1.66$ years, height $=191.62 \pm 5.73 \mathrm{~cm}$, and weight $=86.25 \pm 6.66 \mathrm{~kg}$ ) played in the second league and had at least five years of competitive experience ( $7.5 \pm 2.44$ years). In the first week of the study, when the plyometric training began, the entire team participated in the training. However, due to the injuries and contusions sustained and unforeseen reasons, only 8 players took part in all of the training sessions organised over the course of the sixweek programme, while the remaining players failed to attend at least one of the sessions. The team consisted of 11 players who played in offensive positions ( 3 middle blockers, 5 receivers, and 3 attackers) and 5 players who played in defensive positions ( 3 setters and 2 liberos). All the players were healthy and were qualified for the tournament as they fulfilled the requirements of its organisers. The protocol of the study was approved by the Research Ethics Committee of the University School of Physical Education in Wroclaw. Before participating in the training pro- gramme, the players were informed about the aim of the study and its procedure, and they gave written consent to participation in the study. The homogeneity of the group was assessed based on the standard deviation of the BMI value, which did not exceed $10 \%$ of the arithmetic mean for all of the players $(\mathrm{BMI}=23.47 \pm 1.68)$.

\section{Procedure of the study}

The measurements were performed between October and November, at the beginning of the preparatory period (at 4 weeks) and at the beginning of the competition period (at 2 weeks). The volleyball team trained 5-6 times per week, with each session lasting 90-120 minutes. This included specific volleyball training, practice matches, and regular matches.

The plyometric training was composed of 60-minute sessions that were preceded by specific 10-minute warm-ups.

The warm-ups consisted of the exercises listed below.

- Exercises involving jogging across the sports hall included the following:

- different types of arm swings;

- different types of arm circles;

- forward bounding run with arm swings and circles;

- side step with arm swings and circles.

- Exercises involving marching across the sports hall included the following:

- different types of lunges;

- different types of leg swings;

- different types of bends;

- lunges with arm swings;

- dynamic stretching in place of legs, arms, shoulder girdle, pelvic girdle, and trunk;

- speed training in the form of skipping, multiple jumps, bounds, and accelerations preceded by running in place.

The programme that was implemented (the main part of the plyometric training) consisted of different types of hops and vertical and horizontal jumps performed with varying intensity, speed, and direction. This programme was used twice a week (on Mondays and Wednesdays), and during the remaining days of the week, the players underwent specific volleyball training designed by the coaches and played some practice matches. During the competition period, the players played regular league matches (on Thursdays and Saturdays). All of the players who participated in the study also performed work related to their everyday activity, but, due to its low intensity and irregularity, this work did not have an influence on the training process. The entire plyometric training session lasted from 70 to $90 \mathrm{~min}$ and comprised a specific warm-up, the main part (Tab. 1), and 10 -minute recovery. The recovery part included jogging, static stretching in a standing and sitting position, and rolling of the lower limb muscles.

Table 1. Intensity of the plyometric exercise

\begin{tabular}{|l|c|c|c|l|}
\hline \multicolumn{1}{|c|}{ Exercise/Training 1 } & Intensity & HR & $\begin{array}{c}\text { Movement } \\
\text { direction }\end{array}$ & \multicolumn{1}{|c|}{ Exercise/Training 2 } \\
\hline Bilateral half-squat jumps & high & $\geq 160$ & vertical & Half-squat jumps with heel kick \\
\hline Combination of standing long jump and spiking jump & medium & $140-150$ & mixed & Combination of standing long jump and spiking jump \\
\hline Hurdle hops & high & $\geq 160$ & vertical & Alternate leg vertical box step-ups \\
\hline Split scissor jumps in place & low & $120-140$ & horizontal & Fast bilateral jumps ("frog" jumps) \\
\hline Alternate vertical high knee jumps & high & $\geq 160$ & vertical & Half-squat tuck jumps with knees up \\
\hline
\end{tabular}




\section{Measuring muscle stiffness using MyotonPRO}

The procedure of the study included three measurements performed once a week over 6 consecutive weeks of plyometric training (before the warm-up): in week 0 , week 4 (the effects of the training completed in week 3 ), and week 6 (the effects of the training carried out in week 5).

Each time before the measurement started, the points above particular muscle bellies and attachments were marked (Fig. 1), and the same points were used in all of the measurements. The device was placed perpendicular to the points marked on the skin according to the order shown in Figure 1. Stiffness was measured before the training (before the warm-up) for 15 points.
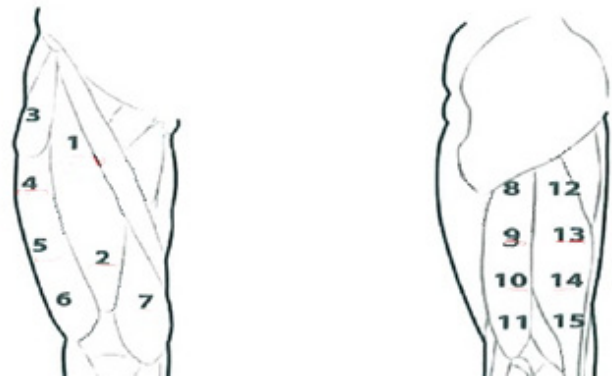

Figure 1. Measurement points in the anterior and posterior thigh muscles

A)

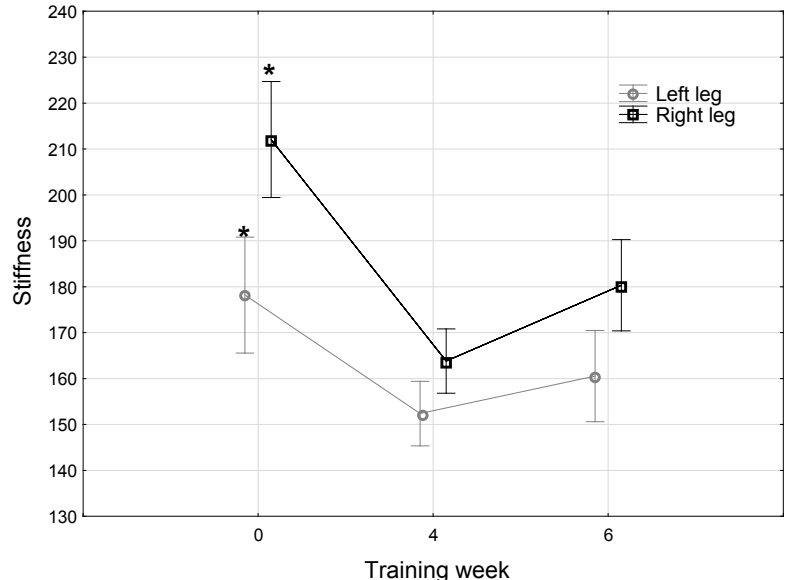

C)

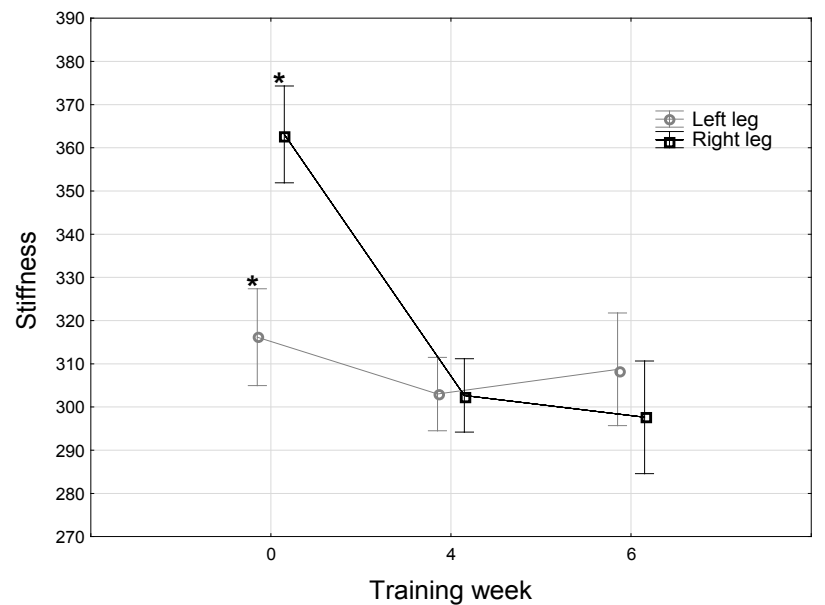

- The measurement points for the anterior thigh muscles were as follows:

- point 1 - upper part of rectus femoris;

- point 2 - lower part of rectus femoris;

- point 3 - tensor fasciae latae;

- point 4 - upper part of vastus lateralis;

- point 5 - middle part of vastus lateralis;

- point 6 - lower part of vastus lateralis;

- point 7 - vastus medialis.

- The measurement points for the posterior thigh muscles were as follows:

- point 8 - upper part of biceps femoris;

- point 9 - middle part of biceps femoris;

- point 10 - middle part of biceps femoris;

- point 11 - lower part of biceps femoris;

- point 12 - upper part of semitendinosus;

- point 13 - middle part of semitendinosus;

- point 14 - middle part of semitendinosus;

- point 15 - lower part of semitendinosus.

The measurements of muscle stiffness lasted approximately 65 minutes. The players came to the assessments in groups of five every 20 minutes, an hour before the plyometric training. The measurement points were identified, and the assessments were performed in a lying position on a physiotherapy table in a room next to the sports hall. The measurements were

B)

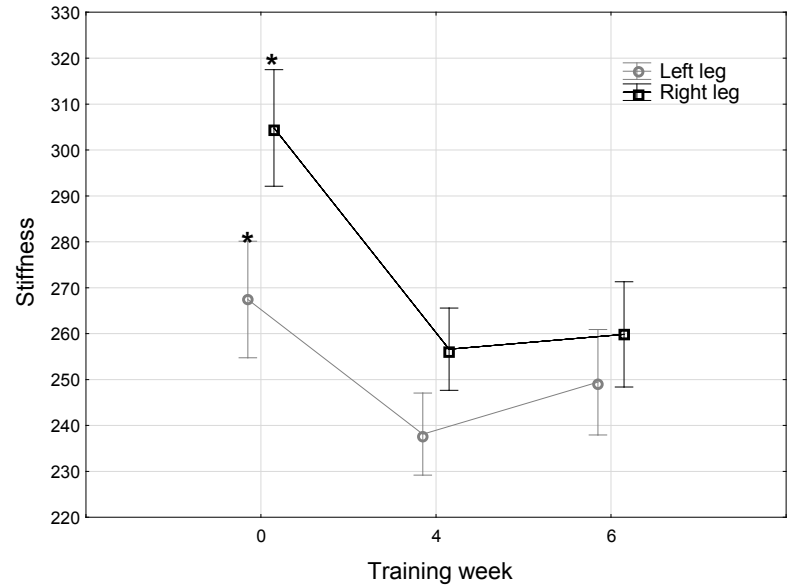

D)

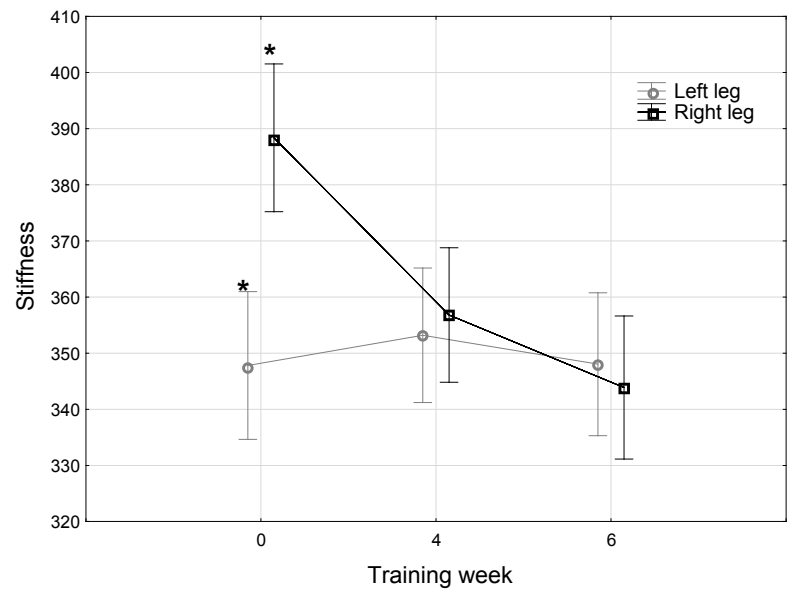

Figure 2. Changes in the stiffness of the semitendinosus muscles of the left and right limbs during six weeks of plyometric training (A - results for point 12, B - results for point 13, C - results for point 14, D - results for point 15; asterisk indicates significant differences between the left and right limbs) 
performed by the same person over six consecutive weeks, so as to avoid errors having to with marking the points and using the measurement device. Individual assessments lasted up to 4 minutes, and the participants underwent them in a random order since this order did not have an impact on the results recorded for muscle stiffness.

\section{Statistical analysis}

The statistical analysis was performed using mean and standard deviation values. The Shapiro-Wilk test showed that the distribution of data for all of the variables examined was normal. The levels of stiffness of particular muscles in the left and right limbs were compared against each other using an unpaired Student t-test, and effect size was estimated using Cohen's $d$ [11]. Effect size was interpreted as insignificant for $\mathrm{d} \leq$ 0.2 , small for $0.2 \leq \mathrm{d} \leq 0.5$, medium for $0.5 \leq \mathrm{d} \leq 0.8$, and large for $0.8 \geq \mathrm{d}$. The statistical analysis was carried out using SPSS version 15.0 (Chicago, USA) for Windows.

\section{Results}

The analysis of stiffness levels in 7 points in the anterior parts and 8 points in the posterior parts of the thigh (Fig. 2) revealed significant differences between the points in the left and right limbs only in the posterior muscles. Significant differences $(\mathrm{p}<0.05)$ were observed for the semitendinosus immediately before the experiment started, whereas in the fourth and sixth training session, the differences were insignificant. In measurement point 12 (Fig. 2A), the difference in muscle stiffness was 33.9 AU before the experiment, 11.4 AU in the fourth week of training, and 19.8 AU in the sixth week of training, whereas in point 13 (Fig. 2B), it was 37.4 AU, 18.5 AU, and 10.4 AU, respectively. In measurement point 14 (Fig. 2C), the difference in muscle stiffness was $46.9 \mathrm{AU}$ before the experiment, $0.3 \mathrm{AU}$ in the fourth week of training, and 11.1 AU in the week of training, while in point 15 (Fig. 2D), it was 40.6 AU, 3.6 AU, and 4.2 AU, respectively.

\section{Discussion}

The programme of assessing muscle stiffness presented in the current study offers new opportunities for the non-invasive examination of the properties of the muscles in athletes exposed to training. It may be an important contribution to the existing body of knowledge, especially that there are several clinical studies examining the parameters of muscle tone and the mechanical properties of the muscles [12-16]; in these studies, the muscles were stimulated mechanically or electrically, and the responses of muscle tissue were analysed.

The results of the measurement in this study have also revealed other interesting facts. The stiffness values recorded for the anterior and posterior thigh muscles may suggest that the adequate selection of loads in plyometric training does not cause disproportions in the stiffness of these muscle groups in consecutive weeks of training. On the contrary, after the training was completed, no significant asymmetries were found in the level of stiffness of the anterior thigh muscles. Previous studies [6] have confirmed that the objective measurement of the tone, tension, and mechanical properties of the muscle, such as its elasticity and stiffness, make it possible to effectively identify overload states and significantly reduce the risk of contusion, which occurs in particular when the training loads have not been adequately chosen.
In addition, we observed statistically significant asymmetry in the stiffness of anterior and posterior thigh muscles before the plyometric training commenced; the values decreased in subsequent weeks of the training, which is a desirable effect [3]. In the case of the current study, it can be surmised that appropriate proportions between the workloads used during training, the competition loads, and active recovery made it possible to increase symmetry in the stiffness of the left and right lower limb muscles, even with high loads imposed on the players' bodies.

Our study revealed significant asymmetry in the stiffness of posterior thigh muscles compared to that of anterior ones. From a physiological point of view [17], the "dynamic-passive" mechanism that is responsible for the stability and mobility of the entire neuromuscular system is constantly receiving feedback concerning the elasticity- and contractility-related properties of the muscle. Muscle stiffness increases stability in the joint, simultaneously increasing the energy cost of this mechanism [18-20]. On the other hand, some authors [21, 22] have reported that an increase in muscle stiffness can be conducive to reducing the energy costs of the above-mentioned mechanism owing to the storage of kinetic energy through the stretching of the muscle and the partial return of the energy when the return motion starts, for example during plyometric training. Taking into account the training macrocycles completed by the players before the study, some probable causes of our findings are the following: a) the inadequate implementation of training loads and the accumulation of competition loads from the previous competition period combined with a lack of proper recovery, b) a lack of physical activity during rest in the transition period before undertaking plyometric training, and c) the difference in technique between the jumps performed as part of plyometric training and those performed using volleyball-specific technique.

The results of the study have confirmed the usefulness of the Myoton Pro device, which makes it possible to quickly and directly assess the level of muscle stiffness after plyometric training. This method may deliver information as to the players' level of fatigue. The use of particular exercise methods and physical loads caused a decrease in the initial differences in the stiffness of the thigh muscles of the two limbs, improving the symmetry between them. Since valuable information has been gained in the study, it seems worth examining the asymmetry in the stiffness of the shoulder girdle and upper limb muscles, which typically work asymmetrically during specific exercise performed in volleyball matches. The measurements of muscle stiffness can be effectively used to assess potential imbalances between the muscles of the two legs and adjust training loads.

Examining athletes of both genders, practising on a professional and amateur level, and exposed to optimal and maximal loads can help determine the range of threshold muscle stiffness values and differences in stiffness asymmetry in particular muscle groups. Exceeding such threshold values could put the players at risk for micro-trauma and, as a result, contusions. The results the measurement of muscle stiffness can be successfully used to assess imbalances between the muscles of the two limbs and correct training loads. Monitoring changes in muscle stiffness after a contusion makes it possible to select an adequate form of physiotherapy and allows for an optimal return to training. The study demonstrated that a six-week plyometric training programme with high loads can improve symmetry in the level of the stiffness of the thigh muscles, which can help prevent contusions and, at the same time, enhance performance. 


\section{Acknowledgements}

We would like to thank the coaches of the team and the volleyball players for participating in the project. The project was financed under a grant awarded by the Polish Ministry of Science and Higher Education for the "Development of University Sports" for the years 2015-2017 (Project No. 04053 N RSA3).

\section{Literature}

1. Wilson G.J., Wood G.A., Elliott B.C. (1991). Optimal stiffness of series elastic component in a stretch-shorten cycle activity. Journal of Applied Physiology 70(2), 825-33.

2. Mullix J., Warner M., Stokes M. (2012). Testing muscle tone and mechanical properties of rectus femoris and biceps femoris using a novel hand held MyotonPRO device: Relative ratios and reliability. Working Papers in Health Sciences 1(1), 1-8.

3. Croisier J.L., Ganteaume S., Binet J., Genty M., Ferret J.M. (2008). Strength imbalances and prevention of hamstring injury in professional soccer players: A prospective study. American Journal of Sports Medicine 36(8), 1469-75.

4. Mooney K., Warner M., Stokes M. (2013). Symmetry and within-session reliability of mechanical properties of biceps brachii muscles in healthy young adult males using the MyotonPRO device. Working Papers in Health Sciences 1(3), $1-11$.

5. Aird L., Samuel D., Stokes M. (2012). Quadriceps muscle tone, elasticity and stiffness in older males: Reliability and symmetry using the MyotonPRO. Archives of Gerontology and Geriatrics 55(2), e31-9.

6. Masi A.T., Nair K., Evans T., Ghandour Y. (2010). Clinical, biomechanical, and physiological translational interpretations of human resting myofascial tone or tension. International Journal of Therapeutic Massage E Bodywork 3(4), 16-28.

7. Brazier J., Bishop C., Simons C., Antrobus M., Read M.J., Turner A.N. (2014). Lower extremity stiffness: Effects on performance and injury and implications for training. Strength and Conditioning Journal 112(3/5), 103-112.

8. Brughelli M., Cronin J. (2008). Influence of running velocity on vertical, leg and joint stiffness: Modelling and recommendations for future research. Sports Medicine 38(8), 647-57.

9. Gunther M., Blickhan R. (2002). Joint stiffness of the ankle and the knee in running. Journal of Biomechanics 35(11), 1459-74.

10. Arampatzis A., Schade F., Walsh M., Brüggemann G.-P. (2001). Influence of leg stiffness and its effect on myodynamic jumping performance. Journal of Electromyography and Kinesiology 11(5), 355-364.
11. Thalheimer W., Cook S. (2002). How to calculate effect sizes from published research: A simplified methodology. Somerville, MA: Work-Learning Research.

12. Chen J.J., Wu Y.N., Huang S.C., Lee H.M., Wang Y.L. (2005). The use of a portable muscle tone measurement device to measure the effects of botulinum toxin type a on elbow flexor spasticity. Archives of Physical Medicine and Rehabilitation 86(8), 1655-60.

13. Gavronski G., Veraksits A., Vasar E., Maaroos J. (2007). Evaluation of viscoelastic parameters of the skeletal muscles in junior triathletes. Physiological Measurement 28(6), 62537.

14. Gennisson J.L., Cornu C., Catheline S., Fink M., Portero P. (2005). Human muscle hardness assessment during incremental isometric contraction using transient elastography. Journal of Biomechanics 38(7), 1543-50.

15. Leonard C.T., Brown J.S., Price T.R., Queen S.A., Mikhailenok E.L. (2004). Comparison of surface electromyography and myotonometric measurements during voluntary isometric contractions. Journal of Electromyography E Kinesiology 14(6), 709-14.

16. Tous-Fajardo J., Moras G., Rodriguez-Jimenez S., Usach R., Doutres D.M., Maffiuletti N.A. (2010). Inter-rater reliability of muscle contractile property measurements using noninvasive tensiomyography. Journal of Electromyography $\mathcal{E}$ Kinesiology 20(4), 761-6.

17. Pearsall A.W.T., Hollis J.M., Russell G.V.Jr., Scheer Z. (2003). A biomechanical comparison of three lower extremity tendons for ligamentous reconstruction about the knee. Arthroscopy 19(10), 1091-6.

18. Riemann B.L., Lephart S.M. (2002). The Sensorimotor System, Part II: The role of proprioception in motor control and functional joint stability. Journal of Athletic Training 37(1), 80-4.

19. Wright V. (1973). Stiffness: A review of its measurement and physiological importance. Physiotherapy 59(4), 107-11.

20. Klinge K., Magnusson S.P., Simonsen E.B., Aagaard P., Klausen K., Kjaer M. (1997). The effect of strength and flexibility training on skeletal muscle electromyographic activity, stiffness, and viscoelastic stress relaxation response. American Journal of Sports Medicine 25(5), 710-6.

21. Gleim G.W., Stachenfeld N.S., Nicholas J.A. (1990). The influence of flexibility on the economy of walking and jogging. Journal of Orthopaedic Research 8(6), 814-23.

22. Craib M.W., Mitchell V.A., Fields K.B., Cooper T.R., Hopewell R., Morgan D.W. (1996). The association between flexibility and running economy in sub-elite male distance runners. Medicine and Science in Sports and Exercise 28(6), $737-43$.

Submitted: October 20, 2017

Accepted: February 14, 2018 